\title{
Copper(II)-Mediated Iodination of 1-Nitroso-2-naphthol
}

\author{
Zarina M. Efimenko ${ }^{1}$, Anton V. Rozhkov ${ }^{1}{ }^{(0}$, Vitalii V. Suslonov ${ }^{1}$, Maxim L. Kuznetsov ${ }^{1,2}{ }^{\mathbb{D}}$, \\ Vadim Yu. Kukushkin ${ }^{1,3}$ (D) and Nadezhda A. Bokach ${ }^{1, *(D)}$ \\ 1 Institute of Chemistry, Saint Petersburg State University, Universitetskaya Nab. 7/9, \\ 199034 Saint Petersburg, Russia; bicbaevazarina@yandex.ru (Z.M.E.); iomcrozhkov@gmail.com (A.V.R.); \\ v.suslonov@spbu.ru (V.V.S.); max@mail.ist.utl.pt (M.L.K.); v.kukushkin@spbu.ru (V.Y.K.) \\ 2 Centro de Química Estrutural, Instituto Superior Técnico, Universidade de Lisboa, 1049-001 Lisbon, Portugal \\ 3 Institute of Chemistry and Pharmaceutical Technologies, Altai State University, 656049 Barnaul, Russia \\ * Correspondence: n.bokach@spbu.ru
}

check for updates

Citation: Efimenko, Z.M.; Rozhkov, A.V.; Suslonov, V.V.; Kuznetsov, M.L.; Kukushkin, V.Y.; Bokach, N.A. Copper(II)-Mediated Iodination of 1-Nitroso-2-naphthol. Molecules 2021, 26, 5708. https://doi.org/10.3390/ molecules 26185708

Academic Editor: Franco Bisceglie

Received: 5 September 2021

Accepted: 17 September 2021

Published: 21 September 2021

Publisher's Note: MDPI stays neutral with regard to jurisdictional claims in published maps and institutional affiliations.

Copyright: (c) 2021 by the authors. Licensee MDPI, Basel, Switzerland. This article is an open access article distributed under the terms and conditions of the Creative Commons Attribution (CC BY) license (https:/ / creativecommons.org/licenses/by/ $4.0 /)$.

\begin{abstract}
The 3-Iodo-1-nitrosonaphthalene-2-ol (I-NON) was obtained by the copper(II)-mediated iodination of 1-nitroso-2-naphthol (NON). The suitable reactants and optimized reaction conditions, providing $94 \%$ NMR yield of I-NON, included the usage of $\mathrm{Cu}(\mathrm{OAc})_{2} \cdot \mathrm{H}_{2} \mathrm{O}$ and 1:2:8 $\mathrm{Cu}$ molar ratio between the reactants. The obtained I-NON was characterized by elemental analyses $(\mathrm{C}$, $\mathrm{H}, \mathrm{N})$, high-resolution $\mathrm{ESI}^{+}$-MS, ${ }^{1} \mathrm{H}$ and ${ }^{13} \mathrm{C}\left\{{ }^{1} \mathrm{H}\right\}$ NMR, FTIR, UV-vis spectroscopy, TGA, and X-ray crystallography (XRD). The copper(II) complexes bearing deprotonated I-NON were prepared as follows: cis-[Cu(I-NON-H)(I-NON)]( $\left.\mathrm{I}_{3}\right)(\mathbf{1})$ was obtained by the reaction between $\mathrm{Cu}(\mathrm{NON}-\mathrm{H})_{2}$ and $\mathrm{I}_{2}$ in $\mathrm{CHCl}_{3} / \mathrm{MeOH}$, while trans-[Cu(I-NON-H $\left.)_{2}\right](2)$ was synthesized from I-NON and $\mathrm{Cu}(\mathrm{OAc})_{2}$ in $\mathrm{MeOH}$. Crystals of trans-[Cu(I-NON-H $\left.)_{2}(\mathrm{THF})_{2}\right](3)$ and trans-[Cu(I-NON-H $\left.)_{2}(\mathrm{Py})_{2}\right](4)$ were precipitated from solutions of 2 in $\mathrm{CHCl}_{3} / \mathrm{THF}$ and $\mathrm{Py} / \mathrm{CHCl}_{3} / \mathrm{MeOH}$ mixtures, respectively. The structures of $\mathbf{1}$ and 3-4 were additionally verified by $\mathrm{X}$-ray crystallography. The characteristic feature of the structures of $\mathbf{1}$ and $\mathbf{3}$ is the presence of intermolecular halogen bonds with the involvement of the iodine center of the metal-bound deprotonated I-NON. The nature of the I $\cdots \mathrm{I}$ and I $\cdots \mathrm{O}$ contacts in the structures of $\mathbf{1}$ and 3, correspondingly, were studied theoretically at the DFT (PBE0-D3BJ) level using the QTAIM, ESP, ELF, NBO, and IGM methods.
\end{abstract}

Keywords: metal-mediated reaction; iodination; copper(II) complexes; nitrosonaphthol; halogen bonding

\section{Introduction}

Nitrosonaphthols, typically existing in the quinone monoxime tautomeric form (see ESI, Scheme S1) [1], represent a group of compounds that found a wide range of useful applications. These species have been applied as precursors in organic synthesis [2], they were used for the analytical determination of bioorganic species [3,4], and they also exhibit some biological activity modes (e.g., carcinogenic) as iminoxyl and hydronitroxide radical source [5]. Nitrosonaphthols with ortho-positioned functional groups, i.e., $\mathrm{NO}(\mathrm{H})$ and $\mathrm{O}(\mathrm{H})$, exhibit chelating properties and they found application as analytical reagents for determination [6-8], extraction [7-9], and sorption [10] of metal ions and also can be applied as sequestering agents for nanoparticle preparation [11]. The $o$-nitrosonaphthols were also used for the preparation of immobilized reagents for optical metal ion sensing [12] and polymer-supported catalysts [13].

A search of synthetically significant routes for the modification and functionalization of nitrosonaphthols could be an important task for the improvement of the analytical properties of these and structurally related species. In particular, initial modification of nitrosonaphthols can include introduction of a halogen atom, e.g., by direct halogenation. The direct chlorination and bromination of 1-nitroso-2-naphthol by the corresponding dihalogens to afford 3-halo-1-nitroso-2-naphthols have been reported [14,15], while the 
iodination is a more complicated task and the iodinated product was obtained only by the exchange reaction of the corresponding brominated derivative [15]. Indeed, the halogenation of aromatic species by $X_{2}$ strongly depends on the identity of a halogen and, in the case of less reactive $I_{2}$, this reaction commonly requires Lewis acid catalysis, e.g., application of copper(II) salts, and it was conducted mainly for electron-rich arenes [16-18] In this work, we report on a novel metal-mediated reaction that proceeds in the system $\mathrm{Cu}^{\mathrm{II}} /$ 1-nitroso-2-naphthol/ $/ \mathrm{I}_{2}$ and leads, after liberation of the ligand, to uncomplexed 3-iodo-1-nitrosonaphthalene-2-ol. We also succeeded in obtaining several copper(II) complexes with the iodinated product, and all our results and findings are detailed in sections that follow.

\section{Results}

\subsection{Synthesis of 3-Iodo-1-nitrosonaphthalen-2-ol}

The 3-Iodo-1-nitrosonaphthalene-2-ol (I-NON) was obtained by the copper(II)mediated iodination of 1-nitroso-2-naphthol (NON). Our initial experiments demonstrated that the copper complex $\left[\mathrm{Cu}(\mathrm{NON}-\mathrm{H})_{2}\right]$ reacted with 8 equivs of molecular iodine in $\mathrm{MeOH}$ to form the $[\mathrm{Cu}(\mathrm{I}-\mathrm{NON}-\mathrm{H})(\mathrm{I}-\mathrm{NON})]\left(\mathrm{I}_{3}\right)(\mathbf{1})$ complex bearing the iodinated ligands (for detailed characterization and relevant discussion, see below). We applied this reaction as a starting point to develop a synthetic route to I-NON (Scheme 1). On the one hand, I-NON, as a representative of the group of nitrosonaphthols, can be useful as a chelator for transition metal complexes and this sequestering can be applied for analytic or extraction purposes $[19,20]$; on the other hand, the iodine functionality in I-NON, acting as a halogen bond $(\mathrm{HaB})$ donor, might be useful for HaB-based crystal engineering [21-25].

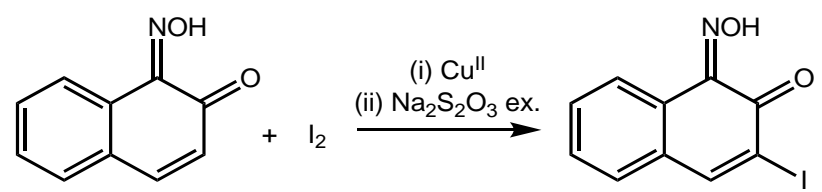

Scheme 1. Synthesis of I-NON via Cu ${ }^{\text {II }}$-mediated iodination of NON.

Our procedure consists, on the first step, in one-pot reaction of NON with a copper(II) salt and $\mathrm{I}_{2}$ followed by, on the second step, the liberation of I-NON by the treatment of the reaction mixture with an excess $\mathrm{Na}_{2} \mathrm{~S}_{2} \mathrm{O}_{3}$. Aiming to develop the two-step synthetic procedure to I-NON, we performed the optimization of the reaction conditions (Table 1) by varying copper(II) and some other metal salts, load of copper(II) salts, and variation of molar ratios between the reactants. We tested several copper(II) sources (Table 1, entries 1-5) for the system $\mathrm{Cu}^{\mathrm{II}} / \mathrm{NON} / \mathrm{I}_{2}\left(1: 2: 2\right.$ molar ratio) and found that the application of $\mathrm{CuCl}_{2} \cdot 2 \mathrm{H}_{2} \mathrm{O}$ gave the highest yield of I-NON (38\%), while the involvement of $\mathrm{Cu}(\mathrm{OAc})_{2} \cdot \mathrm{H}_{2} \mathrm{O}$ produced the best ratio between the product and the unreacted starting materials (2:1). Therefore, for further optimization, we chose $\mathrm{Cu}(\mathrm{OAc})_{2} \cdot \mathrm{H}_{2} \mathrm{O}$. Variation of the $\mathrm{I}_{2}$ load (Table 1 , entries $5-8)$ revealed that the highest yield was achieved with $1: 4 \mathrm{NON} / \mathrm{I}_{2}$ molar ratio. We also attempted the reaction under the catalytic conditions (Table 1 , entries 5 and 9,7 and 10), but observed the I-NON yield drop on decreasing the load of $\mathrm{Cu}(\mathrm{OAc})_{2} \cdot \mathrm{H}_{2} \mathrm{O}$. This experiment indicates that the reaction required the stochiometric amount of copper(II). Other tested metal salts were less effective (Table 1, entry 14) or totally ineffective (Table 1, entries 11-13) in the reaction. Notably, the iodination of NON can proceed under metal-free conditions (Table 1, entry 15), but, in contrast to the optimized $\mathrm{Cu}^{\mathrm{II}}$-mediated reaction, this synthesis gave only ca. $24 \%$ conversion to I-NON. An increase of the reaction time to $3 \mathrm{~d}$ did not affect the conversion of the starting materials. In summary, the results of these tests demonstrated that the optimal conditions for this copper(II)-involving reaction included the usage of $\mathrm{Cu}(\mathrm{OAc})_{2} \cdot \mathrm{H}_{2} \mathrm{O}$ and 1:2:8 $\mathrm{Cu}^{\mathrm{II}} / \mathrm{NON} / \mathrm{I}_{2}$ molar ratios between the reactants. Although the reaction, as we found, could be carried out without the metal source (ca. $25 \%$ conversion, as verified by GC-MS monitoring; the isolation was not performed), the 
employment of copper(II) led to nearly quantitative conversion of NON to I-NON (94\%; entry 8).

Table 1. Optimization of the reaction conditions. ${ }^{1}$.

\begin{tabular}{|c|c|c|c|c|c|}
\hline \multirow[b]{2}{*}{ Entry } & \multicolumn{3}{|c|}{ Conditions } & \multicolumn{2}{|c|}{ Product Identification } \\
\hline & M Salt & $\begin{array}{c}\text { M/NON } \\
\text { Ratio }\end{array}$ & $\begin{array}{c}\mathrm{I}_{2} / \mathrm{NON} \\
\text { Ratio }\end{array}$ & $\begin{array}{c}\text { I-NON/ } \\
\text { NON Ratio }\end{array}$ & $\begin{array}{c}\text { Yield of } \\
\text { I-NON (\%) } \\
\left({ }^{1} \mathrm{H} \text { NMR) }\right.\end{array}$ \\
\hline 1 & $\mathrm{CuCl}_{2} \cdot 2 \mathrm{H}_{2} \mathrm{O}$ & $1 / 2$ & $1 / 1$ & $1.15 / 1$ & 38 \\
\hline 2 & $\mathrm{Cu}\left(\mathrm{SO}_{4}\right)_{2} \cdot 5 \mathrm{H}_{2} \mathrm{O}$ & $1 / 2$ & $1 / 1$ & $1 / 1$ & 32 \\
\hline 3 & $\mathrm{Cu}(\mathrm{acac})_{2}$ & $1 / 2$ & $1 / 1$ & $1.7 / 1$ & 6 \\
\hline 4 & $\mathrm{Cu}\left(\mathrm{NO}_{3}\right)_{2} \cdot 2 \mathrm{H}_{2} \mathrm{O}$ & $1 / 2$ & $1 / 1$ & 1.14 & 21 \\
\hline 5 & $\mathrm{Cu}(\mathrm{OAc})_{2} \cdot \mathrm{H}_{2} \mathrm{O}$ & $1 / 2$ & $1 / 1$ & $2 / 1$ & 18 \\
\hline 6 & $\mathrm{Cu}(\mathrm{OAc})_{2} \cdot \mathrm{H}_{2} \mathrm{O}$ & $1 / 2$ & $2 / 1$ & $3.5 / 1$ & 30 \\
\hline 7 & $\mathrm{Cu}(\mathrm{OAc})_{2} \cdot \mathrm{H}_{2} \mathrm{O}$ & $1 / 2$ & $3 / 1$ & $16 / 1$ & 81 \\
\hline 8 & $\mathrm{Cu}(\mathrm{OAc})_{2} \cdot \mathrm{H}_{2} \mathrm{O}$ & $1 / 2$ & $4 / 1$ & $13.5 / 1$ & 94 \\
\hline 9 & $\mathrm{Cu}(\mathrm{OAc})_{2} \cdot \mathrm{H}_{2} \mathrm{O}$ & $1 / 4$ & $1 / 1$ & $0.04 / 1$ & 2.6 \\
\hline 10 & $\mathrm{Cu}(\mathrm{OAc})_{2} \cdot \mathrm{H}_{2} \mathrm{O}$ & $1 / 4$ & $3 / 1$ & $2.7 / 1$ & 53 \\
\hline 11 & $\mathrm{CuI}$ & $1 / 2$ & $4 / 1$ & $-/ 1$ & - \\
\hline 12 & $\mathrm{Ni}(\mathrm{OAc})_{2} \cdot 4 \mathrm{H}_{2} \mathrm{O}$ & $1 / 2$ & $4 / 1$ & $0.31 / 1$ & $<1$ \\
\hline 13 & $\mathrm{Co}(\mathrm{OAc})_{2} \cdot 4 \mathrm{H}_{2} \mathrm{O}$ & $1 / 2$ & $4 / 1$ & $-/ 1$ & - \\
\hline 14 & $\mathrm{FeCl}_{3} \cdot 6 \mathrm{H}_{2} \mathrm{O}$ & $1 / 2$ & $4 / 1$ & $2 / 1$ & 23 \\
\hline 15 & -2 & - & $2 / 1$ & $1 / 1.5$ & 24 \\
\hline
\end{tabular}

${ }^{1}$ Typical reaction conditions: (1) Metal salt $(0.08-0.15 \mathrm{mmol}), 1$-nitroso-2-naphthol $(0.3 \mathrm{mmol})$, and molecular iodine $(0.3-1.2 \mathrm{mmol})$ in $\mathrm{CHCl}_{3} / \mathrm{MeOH}(1 / 1 \mathrm{v} / \mathrm{v} ; 30 \mathrm{~mL})$, at RT overnight, whereupon the solvent was evaporated to dryness; (2) The residue formed was dissolved in THF $(30 \mathrm{~mL})$ and treated with a saturated aqueous solution of $\mathrm{Na}_{2} \mathrm{~S}_{2} \mathrm{O}_{3}(25 \mathrm{~mL})$. Organic layer was dried over $\mathrm{MgSO}_{4}$ and then the solvent was removed in vacuo at $30-35{ }^{\circ} \mathrm{C}$ to give the crude products, which were analyzed by ${ }^{1} \mathrm{H}$ NMR. ${ }^{2}$ After keeping for $24 \mathrm{~h}$, the system was analyzed by liquid chromatography.

Based on all these observations, one can conclude that the iodination of NON proceeded in the coordination sphere of $\mathrm{Cu}^{\mathrm{II}}$, while the optimal reactant ratio $\mathrm{Cu}^{\mathrm{II}} / \mathrm{NON}$ (1:2) corresponded to the coordination of NON to give $\left\{\mathrm{Cu}(\mathrm{NON}-\mathrm{H})_{2}\right\}$ species, which then converted to the iodination product, bearing the $\left\{\mathrm{Cu}(\mathrm{I}-\mathrm{NON}-\mathrm{H})_{2}\right\}$ moiety. Therefore, $\mathrm{Na}_{2} \mathrm{~S}_{2} \mathrm{O}_{3}$ on the second step of the synthetic scheme can play the dual role: It was required for the neutralization of the excess of $\mathrm{I}_{2}$ and, simultaneously, it liberated I-NON from the formed complex via the $\mathrm{Cu}^{\mathrm{II}}-$-to- $\mathrm{Cu} \mathrm{u}^{\mathrm{I}}$ reduction, followed by the complexation of the latter with $\mathrm{I}^{-}$and the precipitation of $\mathrm{CuI}$.

After the optimization, we also attempted to extend this copper(II)-mediated reaction to other substrates, such as 2-nitroso-1-naphthol or 1,2-nitrosophenol; however, only mixtures of yet unidentified products were obtained and any iodination product was not detected by ${ }^{1} \mathrm{H}$ NMR or HRESI ${ }^{+}$-MS.

The structure of I-NON was studied by single-crystal X-ray diffractometry (XRD, Figure 1). Inspection of bond lengths' distribution (N1-O1 1.368(11), N1-C1 1.296(13), C2-O2 1.225(13) $\AA$ ) confirmed that I-NON existed in the quinone monoxime form in a solid state, similarly to solid NON [26]. Each of two neighboring molecules of I-NON

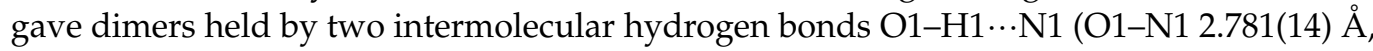
$\left.\angle \mathrm{O} 1-\mathrm{H} 1-\mathrm{N} 1132.9(6)^{\circ}\right)($ Figure S18, ESI). Molecules were also connected via Type I $[21,27]$ I $\cdots$ I contacts (I1I1 3.7550(16) $\left.\AA, \angle I 1-I 1-C 3 ~ 162.7(3)^{\circ}\right)$. For characterization of I-NON, see the ESI. 


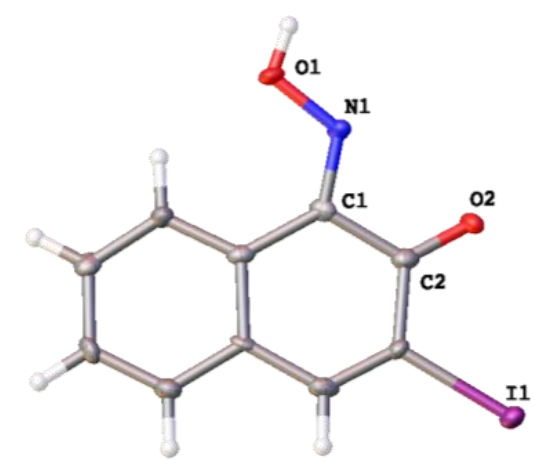

Figure 1. View of the molecular structure of I-NON obtained from XRD study. Nonhydrogen atoms are given as probability ellipsoids of atomic displacements $(p=0.5)$.

Thus, we developed a simple and facile approach to I-NON starting from NON. We found only one study focused on the synthesis of I-NON. The latter included the reaction of 3-bromo-1-nitrosonaphthalen-2-ol (Br-NON) with excess KI to give I-NON in $60 \%$ yield [28]. In turn, Br-NON can be obtained by the bromination of NON with $\mathrm{Br}_{2}$ [29]. Our procedure gave a significantly higher yield of I-NON (94\%) and did not require the usage of the harmful $\mathrm{Br}_{2}$.

We verified the role of metal center control on the reactivity of the deprotonated NON ligand as compared to uncomplexed NON, which is, at least, dual. Firstly, it was protecting the oxime nitrogen from the well-known oxidative deoximation (ref. [30] and references therein) and, secondly, the copper site stabilized the oxo(1-)-substituent in the benzene ring directing the iodination in the ortho-position. Conventional activation of $\mathrm{I}_{2}$ by a Lewis acid should also be taken into account.

\subsection{Copper(II) Complexes Featuring 3-Iodo-1-nitrosonaphthalen-2-ol}

I-NON, as the structural analog of NON, can be also applied as a ligand for metal binding. We studied the ligand properties of I-NON and thus synthesized two I-NONbased $\mathrm{Cu}^{\mathrm{II}}$ complexes. The complex trans-[Cu(I-NON-H $\left.)_{2}\right](2)$ was obtained by the reaction of I-NON with $\mathrm{Cu}(\mathrm{OAc})_{2} \cdot \mathrm{H}_{2} \mathrm{O}$ in $\mathrm{MeOH}$ and it was isolated as a black solid, while cis$[\mathrm{Cu}(\mathrm{I}-\mathrm{NON}-\mathrm{H})(\mathrm{I}-\mathrm{NON})]\left(\mathrm{I}_{3}\right)(\mathbf{1})$ was generated by the treatment of $\left[\mathrm{Cu}(\mathrm{NON}-\mathrm{H})_{2}\right]$ with 8 -fold excess of $\mathrm{I}_{2}$ in a $\mathrm{CHCl}_{3} / \mathrm{MeOH}$ solution at RT. For details of characterization of $\mathbf{1}$ and 2, see ESI.

Crystallization of 2 from the $\mathrm{MeOH} / \mathrm{THF}$ solution resulted in the release of trans-[Cu(I$\left.\mathrm{NON}-\mathrm{H})_{2}(\mathrm{THF})_{2}\right](3)$, while the crystallization of trans $-\left[\mathrm{Cu}(\mathrm{I}-\mathrm{NON}-\mathrm{H})_{2}\right]$ from $\mathrm{MeOH} / \mathrm{Py}$ led to the precipitation of crystals of trans-[Cu(Py) $\left.{ }_{2}(\mathrm{I}-\mathrm{NON}-\mathrm{H})_{2}\right](4)$. Complex 1 was crystallized directly from the reaction mixture. Structures of these three complexes were studied by XRD (Figures 2-4, Table 2).

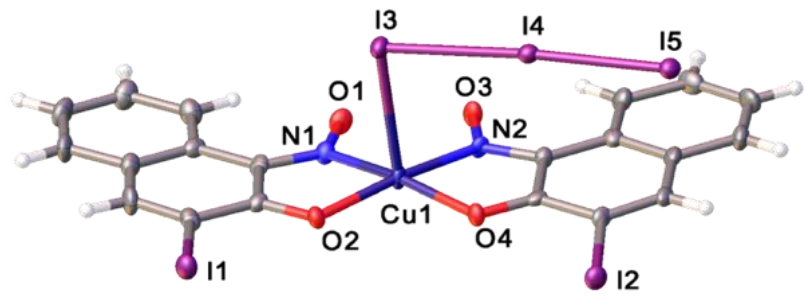

Figure 2. View of the molecular structure of 1 obtained from XRD study. Nonhydrogen atoms are given as probability ellipsoids of atomic displacements $(p=0.5)$. 


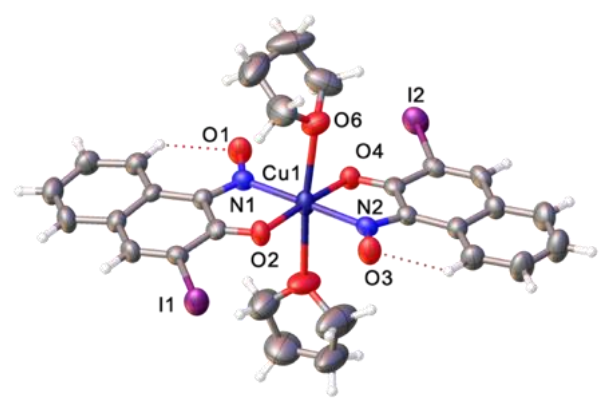

Figure 3. View of the molecular structure of 3 obtained from XRD study. Only one from two symmetrical, independent molecules 3 is given for the sake of simplicity. Nonhydrogen atoms are given as probability ellipsoids of atomic displacements $(p=0.5)$.

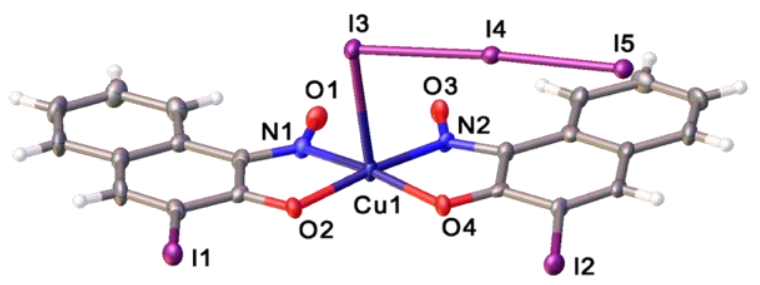

Figure 4. View of the molecular structure of 4 obtained from XRD study. Nonhydrogen atoms are given as probability ellipsoids of atomic displacements $(p=0.5)$.

Table 2. Selected bond lengths for the $\mathrm{Cu}^{\mathrm{II}}$ complexes.

\begin{tabular}{|c|c|c|c|}
\hline \multirow{2}{*}{ Bond } & \multicolumn{3}{|c|}{ Bond Length, Å } \\
\hline & 1 & 3 & 4 \\
\hline \multirow{5}{*}{$\mathrm{Cu}-\mathrm{N}$} & & $1.964(5)$ & \multirow{5}{*}{$2.018(4)$} \\
\hline & 1.963(5) & $1.967(5)$ & \\
\hline & $1.955(5)$ & $1.969(5)$ & \\
\hline & & $1.977(5)$ & \\
\hline & & 1.953(4) & \\
\hline \multirow{4}{*}{$\mathrm{Cu}-\mathrm{O}$} & $1.954(4)$ & $1.955(4)$ & \multirow{4}{*}{$2.302(3)$} \\
\hline & $1.976(4)$ & $1.954(4)$ & \\
\hline & & $1.957(4)$ & \\
\hline & & $1.260(5)$ & \\
\hline \multirow{4}{*}{$\mathrm{N}-\mathrm{O}$} & $1.299(7)$ & $1.268(5)$ & \multirow{4}{*}{$1.282(5)$} \\
\hline & $1.330(7)$ & $1.240(6)$ & \\
\hline & & $1.240(6)$ & \\
\hline & & $1.339(7)$ & \\
\hline \multirow{4}{*}{$\mathrm{N}-\mathrm{C}$} & $1.313(8)$ & $1.343(6)$ & \multirow{3}{*}{$1.341(5)$} \\
\hline & $1.313(8)$ & $1.354(6)$ & \\
\hline & & $1.348(6)$ & \\
\hline & & $1.263(6)$ & \multirow{5}{*}{$1.236(5)$} \\
\hline \multirow{4}{*}{$\mathrm{O}-\mathrm{C}$} & $1.280(8)$ & $1.269(6)$ & \\
\hline & $1.249(8)$ & $1.271(6)$ & \\
\hline & & $1.270(6)$ & \\
\hline & & $2.535(5)$ & \\
\hline \multirow{3}{*}{$\mathrm{Cu}-\mathrm{O}_{\mathrm{THF}}$} & & $2.448(4)$ & \multirow{5}{*}{$2.028(4)$} \\
\hline & & $2.473(6)$ & \\
\hline & & $2.514(6)$ & \\
\hline $\mathrm{Cu}-\mathrm{N}_{\mathrm{Py}}$ & & & \\
\hline $\mathrm{Cu}-\mathrm{I}$ & $2.9417(8)$ & & \\
\hline
\end{tabular}


The crystal structure of $\mathbf{3}$ contains two symmetrical, independent molecules of 3 . The structure of 4 consists of independent halves of the complex molecule, while the structure of $\mathbf{1}$ contains molecules of one type. The structure of $\mathbf{1}$ demonstrates the cis$(N, N)$, cis- $(O, O)$ geometry, while 3 and 4 exist in the trans- $(N, N)$,trans- $(O, O)$ form. In $\mathbf{1}$, the copper(II) center forms two $\mathrm{Cu}-\mathrm{N}$, two $\mathrm{Cu}-\mathrm{O}$, and one $\mathrm{Cu}-\mathrm{I}$ coordination bonds, being in the square-pyramidal environment.

The Cu-I distance (2.9417(8) $\AA$ ) is slightly longer than those typical for the conventional $\mathrm{Cu}-\mathrm{I}$ bonds (2.68 $\AA$ ) [31] and exhibits semicoordinative [32] nature. In 3, copper(II) is in a distorted octahedral environment, where the equatorial $\mathrm{Cu}-\mathrm{N}(1.964(5)-1.977(5) \AA)$ and $\mathrm{Cu}-\mathrm{O}\left(1.953(4)-1.957(4) \AA\right.$ ) bonds are distinctly shorter than the axial $\mathrm{Cu}-\mathrm{O}_{\mathrm{THF}}$ bonds (2.448(4)-2.535(5) $\AA$ ). In 4, the coordination octahedron is less distorted with quite similar distances of the $\mathrm{Cu}-\mathrm{N}(2.018(4) \AA)$ and $\mathrm{Cu}-\mathrm{N}_{\text {Py }}(2.028(4) \AA)$ bonds and with a slightly

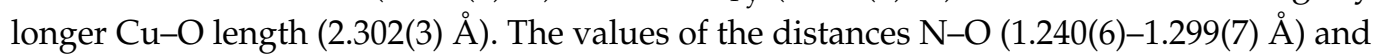
$\mathrm{N}-\mathrm{C}(1.313(8)-1.354(6) \AA)$ of the I-NON-H ligands in 3 and 4 and one I-NON-H ligand in 1 support the existence of these ligands predominantly in the nitrosophenolate form. In contrast, the ligated I-NON in 1 demonstrates the interatomic separations (N-O 1.330(7), $\mathrm{N}-\mathrm{C} 1.313(8)$, and C-O 1.249(8) $\AA$ ), which are more typical for the quinone monoxime form.

Several intermolecular noncovalent interactions were identified in the structures of 1 and 3-4 (Table 3). In 1, the $\mathrm{Cu}$ center forms the semicoordinative bond with an I atom of the $\mathrm{I}_{3}{ }^{-}$ligand of neighboring complex molecule. The $\mathrm{Cu} 1-\mathrm{I} 4$ distance $(3.59 \AA)$ is greater than the corresponding sum of Bondi van der Waals radii [33,34] (3.38 $\AA$ ), but is still substantially smaller than sum of Batsanov crystallographic van der Waals radii (4.1 ̊) [35], and, therefore, the Cu1-I4 linkage can be attributed to the semicoordination bond [32].

Table 3. Selected noncovalent interactions in the $\mathrm{Cu}^{\mathrm{II}}$ complexes.

\begin{tabular}{|c|c|c|c|}
\hline $\begin{array}{c}\text { Contacting } \\
\text { Atoms }\end{array}$ & Distance, $\AA\left(\mathrm{N}_{\mathrm{c}}\right)^{1}$ & Angle, ${ }^{\circ}$ & $\begin{array}{c}\text { Contact } \\
\text { Type/Name }\end{array}$ \\
\hline \multicolumn{4}{|c|}{1} \\
\hline $\mathrm{Cu} 1 \cdots \mathrm{I} 4$ & $3.5935(8)(1.06)$ & Cu1-I4-I5 88.225(17) & semicoordination \\
\hline $\mathrm{I} 1 \cdots \mathrm{I} 3$ & $3.9656(6)(1.00)$ & $\begin{array}{l}\text { C3-I1-I3 178.76(17) } \\
\text { I1-I3-Cu1 97.06(2) }\end{array}$ & $\mathrm{HaB}(\mathrm{I} \cdots \mathrm{I}) 1$ \\
\hline $\mathrm{I} 2 \cdots \mathrm{I} 4$ & $3.7872(6)(0.95)$ & $\begin{array}{l}\text { C-I2-I4 150.6(1) } \\
\text { I2-I4-I5 101.229(17) } \\
3\end{array}$ & $\mathrm{HaB}(\mathrm{I} \cdots \mathrm{I}) 2$ \\
\hline $\mathrm{I} 2 \cdots \mathrm{O} 1$ & $3.159(4)(0.90)$ & $\begin{array}{c}\text { C13-I2-O1 } 152.33(15) \\
\text { I2-O1-N1 156.7(3) }\end{array}$ & $(\mathrm{I} \cdots \mathrm{O}) 1$ \\
\hline $\mathrm{I} 1 \cdots \mathrm{O} 3$ & $3.146(4)(0.90)$ & $\begin{array}{c}\text { C3-I1-O3 150.64(16) } \\
\text { I1-O3-N2 152.2(3) }\end{array}$ & $(\mathrm{I} \cdots \mathrm{O}) 2$ \\
\hline I2A $\cdots \mathrm{O} 3 \mathrm{~A}$ & $3.187(4)(0.91)$ & $\begin{array}{c}\mathrm{C} 13 \mathrm{~A}-\mathrm{I} 2 \mathrm{~A}-\mathrm{O} 3 \mathrm{~A} \\
152.97(12) \\
\mathrm{I} 2 \mathrm{~A}-\mathrm{O} 3 \mathrm{~A}-\mathrm{N} 2 \mathrm{~A} 160.9(3)\end{array}$ & $(\mathrm{I} \cdots \mathrm{O}) 3$ \\
\hline I1A $\cdots$ O1A & $3.566(4)(1.02)^{2}$ & $\begin{array}{l}\text { C3A-I1A-O1A 138.07(16) } \\
\text { I1A-O1A-N1A 142.7(3) }\end{array}$ & $(\mathrm{I} \cdots \mathrm{O}) 4$ \\
\hline
\end{tabular}

${ }^{1}$ The normalized contact $\left(\mathrm{N}_{\mathrm{c}}\right)$ is defined as the ratio between the separation observed in the crystal and the sum of Bondi vdW radii of interacting atoms: $\mathrm{Nc}=\mathrm{d} / \sum_{\mathrm{vdW}} ; \sum_{\mathrm{vdW}}(\mathrm{I}+\mathrm{Cu})=3.38 \AA, \sum_{\mathrm{vdW}}(\mathrm{I}+\mathrm{I})=3.96 \AA$, $\sum_{\mathrm{vdW}}(\mathrm{I}+\mathrm{I})=3.50 \AA .{ }^{2}$ Despite $\mathrm{N}_{\mathrm{c}}>1$, the results of our calculations support the existence of noncovalent bonding.

\subsection{Halogen Bonding Interactions Involving Ligated I-NON}

Organoiodine atoms from I-NON-H and I-NON ligands form HaBs [21] with iodine atoms of the $\mathrm{I}_{3}{ }^{-}$ligands. In 3, I ... H HaBs were detected between I-NON-H ligands. In all structures, several intermolecular hydrogen bonds, e.g., $\mathrm{H}_{\text {aryl }} \cdots \mathrm{O}, \mathrm{H}_{\text {aryl }} \cdots \mathrm{I}$, were also recognized. In view of the importance of $\mathrm{HaB}$ interactions in crystal engineering and to 
verify s-hole properties of the ligated I-NON, we studied HaBs in the structures of $\mathbf{1}$ and 3 in more detail by computational methods.

The nature of the HaBs in $\mathbf{1}$ and $\mathbf{3}$ was studied theoretically at the DFT (PBE0-D3BJ) level using the quantum theory of atoms in molecules (QTAIM), electrostatic potential (ESP), electron localization function (ELF), natural bond orbital (NBO), and independent gradient model (IGM) analyses. The calculations were carried out for bimolecular clusters BM1 $\mathbf{1}_{1}$, $\mathbf{B M 1}_{2}, \mathbf{B M}_{1}, \mathbf{B M}_{2}$, and $\mathbf{B M}_{3}$ with geometries corresponding to the XRD structures of 1 and 3 (Figure 5, see Computational Details). In 1, two I ..I HaBs formed by the organoiodine atoms of the I-NON-H and I-NON ligands were recognized, i.e., $\mathrm{HaB}(\mathrm{I} \cdots \mathrm{I}) 1$ and $\mathrm{HaB}(\mathrm{I} \cdots \mathrm{I}) 2$ (Table 3). The former bond clearly belongs to type II [27] interactions, with

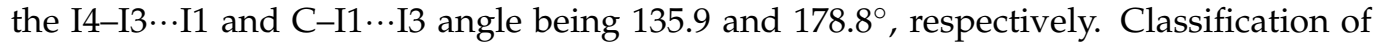
$\mathrm{HaB}(\mathrm{I} \cdots \mathrm{I}) 2$ based on geometrical parameters is not so straightforward since the $\mathrm{C}-\mathrm{I} 2 \cdots \mathrm{I} 4$ angle is $150.6^{\circ}$. However, the ESP, ELF, and NBO analyses clearly indicate that this bond is also of type II with the I2 atom serving as an $\mathrm{HaB}$ donor. The low value of the C-I2 $\cdots \mathrm{I} 4$ angle is accounted for by packing effects, which force the deviation of this HaB from the optimal directionality. In 3, four I $\cdots \mathrm{O}$ bondings were detected, i.e., $\mathrm{HaB}(\mathrm{I} \cdots \mathrm{O}) 1-\mathrm{HaB}(\mathrm{I} \cdots \mathrm{O}) 4$ (the $\mathrm{C}-\mathrm{I} \cdots \mathrm{O}$ and $\mathrm{N}-\mathrm{O} \cdots \mathrm{I}$ angles being $138.1-153.0^{\circ}$ and $142.7-160.9^{\circ}$, respectively). Geometry of these interactions resembles the halogen $\cdots$ halogen type I [27] interactions.
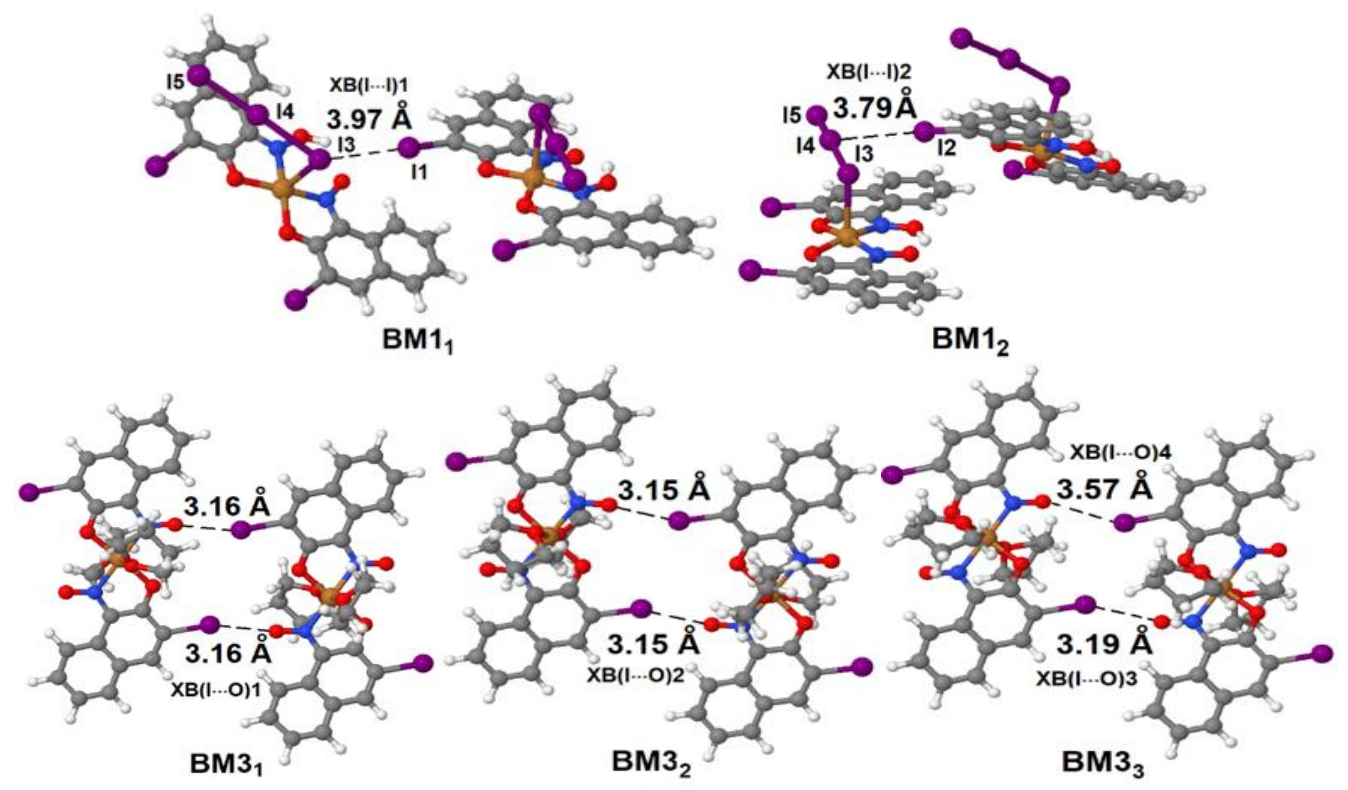

Figure 5. Computational models.

The QTAIM analysis revealed bond critical points (BCPs) for all these contacts (Figure 6). The calculated values of electron density, $\rho_{\mathrm{b}}$, its Laplacian, $\nabla^{2} \rho_{\mathrm{b}}$, potential, and kinetic energy densities $\left(\mathrm{V}_{\mathrm{b}}\right.$ and $\left.\mathrm{G}_{\mathrm{b}}\right)$ at the $\mathrm{BCP}$ are typical for halogen bonds of weak-to-medium strength [36-39] (Table 4). These parameters are lower for $\mathrm{HaB}(\mathrm{I} \cdots \mathrm{I}) 1$ and $\mathrm{HaB}(\mathrm{I} \cdots \mathrm{O}) 4$ correlating with the longer I $\cdots \mathrm{I}$ and $\mathrm{I} \cdots \mathrm{O}$ contacts in these bonds. Meanwhile, all these bondings have an attractive nature, as indicated by the negative sign of the second eigenvalue of the Hessian matrix at the BCP, $\lambda_{2, b}$ (Table 4), and confirmed by the IGM plots of the $\operatorname{sign}\left(\lambda_{2}\right) \rho(\mathrm{r})$ function mapped on the isosurface of the $\delta g^{\text {inter }}$ descriptor (Figure 6). 

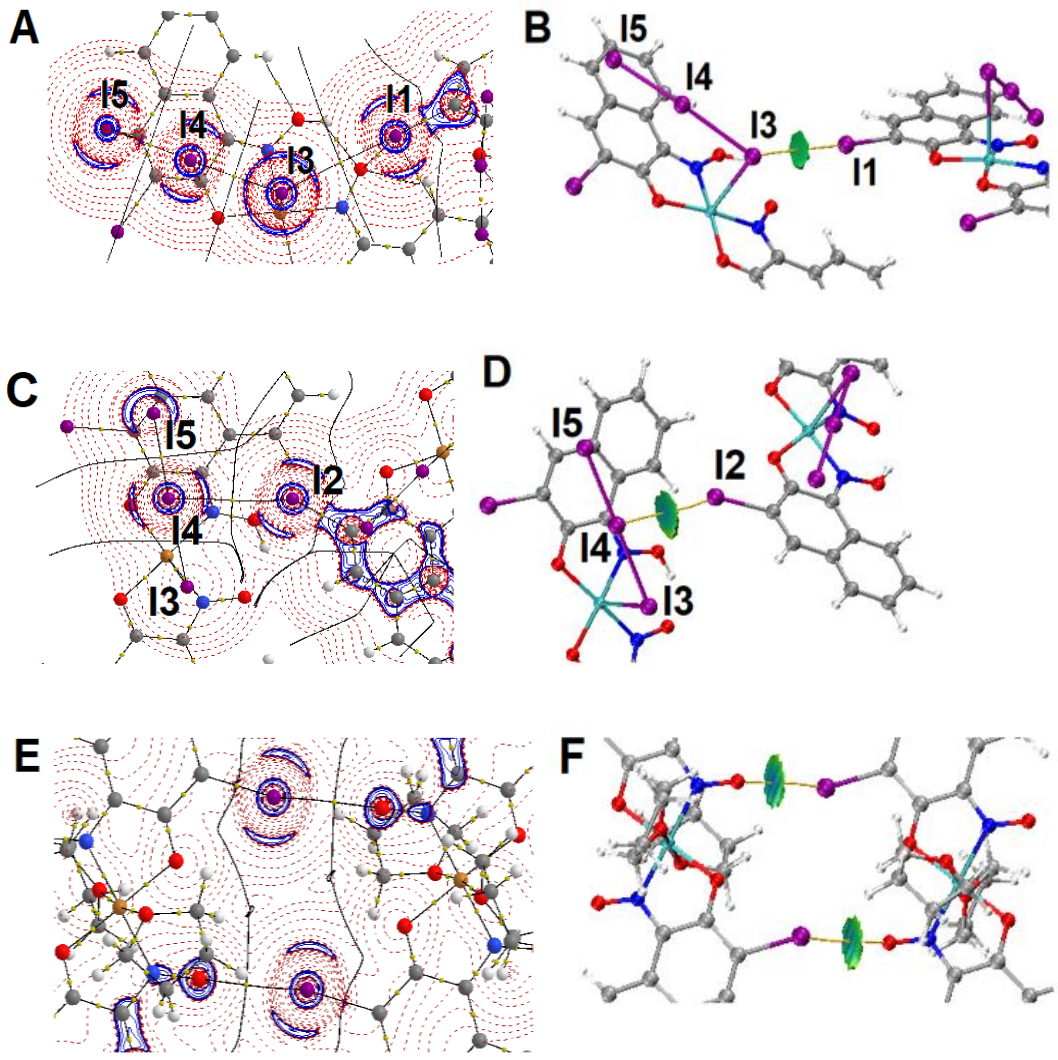

Figure 6. Contour line diagram of the Laplacian distribution $\nabla^{2} \rho(\mathrm{r})$, selected bond paths, zero flux surfaces $(\mathbf{A}, \mathbf{C}, \mathbf{E})$ and the $\operatorname{sign}\left(\lambda_{2}\right) \rho(\mathrm{r})$ function mapped on the $\delta g^{\text {inter }}$ isosurface of 0.005 a.u. and blue-cyan-green-yellow-red color scale $-0.02<\operatorname{sign}\left(\lambda_{2}\right) \rho(\mathrm{r})<0.02$ for the I $\cdots \mathrm{I}$ and $\mathrm{I} \cdots \mathrm{O}$ contacts $(\mathrm{B}, \mathrm{D}, \mathrm{F})$ for $\mathrm{BM1}_{1}(\mathrm{~A}, \mathrm{~B}), \mathrm{BM1}_{2}(\mathrm{C}, \mathrm{D})$ and $\mathrm{BM} 3_{1}(\mathrm{E}, \mathrm{F})$.

Table 4. Halogen bond lengths, $d$ (in $\AA$ ), calculated electron density, $\rho_{\mathrm{b}}$, its Laplacian, $\nabla^{2} \rho_{\mathrm{b}}$, potential and kinetic energy densities, $V_{b}$ and $G_{b}$, second eigenvalue of the hessian matrix, $\lambda_{2, b}$, at BCPs (in a.u.) and interaction energies, $E_{\text {int }}$ (in $\mathrm{kcal} / \mathrm{mol}$ ).

\begin{tabular}{cccccccccc}
\hline Cluster & $\mathbf{H a B}$ & $\mathbf{d}(\mathbf{I} \ldots \mathbf{I} / \mathbf{O})$ & $\boldsymbol{\rho}_{\mathbf{b}}$ & $\nabla^{2} \boldsymbol{\rho}_{\mathbf{b}}$ & $\mathbf{V}_{\mathbf{b}}$ & $\mathbf{G}_{\mathbf{b}}$ & $\boldsymbol{\lambda}_{\mathbf{2}, \mathbf{b}}$ & $\mathbf{E}_{\text {int }}(\mathbf{B S S E})$ & $\mathbf{E}_{\text {int }}(\mathbf{S})$ \\
\hline $\mathbf{B M 1}_{\mathbf{1}}$ & $(\mathrm{I} \cdots \mathrm{I}) 1$ & 3.966 & 0.0058 & 0.0191 & -0.0022 & 0.0035 & -0.0026 & -5.1 & -9.0 \\
$\mathbf{B M 1}_{\mathbf{2}}$ & $(\mathrm{I} \cdots \mathrm{I}) 2$ & 3.787 & 0.0089 & 0.0290 & -0.0039 & 0.0056 & -0.0046 & -9.2 & -10.4 \\
$\mathbf{B M 3}_{1}$ & $(\mathrm{I} \cdots \mathrm{O}) 1$ & 3.159 & 0.0095 & 0.0458 & -0.0063 & 0.0089 & -0.0059 & -4.0 & -7.2 \\
$\mathbf{B M 3}_{2}$ & $(\mathrm{I} \cdots \mathrm{O}) 2$ & 3.146 & 0.0100 & 0.0471 & -0.0065 & 0.0091 & -0.0065 & -4.0 & -6.9 \\
$\mathbf{B M 3}_{3}$ & $(\mathrm{I} \cdots \mathrm{O}) 3$ & 3.187 & 0.0089 & 0.0430 & -0.0057 & 0.0082 & -0.0058 & $-7.23^{1}$ & -7.7 \\
$\mathbf{B M}_{3}$ & $(\mathrm{I} \cdots \mathrm{O}) 4$ & 3.566 & 0.0046 & 0.0191 & -0.0023 & 0.0035 & -0.0026 & & -5.4 \\
\hline
\end{tabular}

${ }^{1}$ Total interaction energy of both $\mathrm{I} \cdots \mathrm{O}$ bonds in $\mathbf{B M}_{3}$.

Formation of type II HaB may be interpreted as a result of the electrostatic interaction between the region of the negative ESP around the HaB acceptor atom and the region of the positive ESP ( $\sigma$-hole) at the HaB donor atom. Indeed, in the optimized structure of the monomer cis- $[\mathrm{Cu}(\mathrm{I}-\mathrm{NON}-\mathrm{H})(\mathrm{I}-\mathrm{NON})]\left(\mathrm{I}_{3}\right)(\mathbf{M 1})$, the maximum values of ESP at the terminal ends of the $\mathrm{C}-\mathrm{I}$ bonds calculated on the $1.1 \times$ van der Waals surface are 0.054 and 0.061 a.u. (Figure 7A). Despite the C-I2 $\cdots$ I4 angle in 1 is significantly lower than $180^{\circ}$, a belt of the negative ESP around the I4 atom is still directed towards the $\sigma$-hole of I2 providing the electrostatic interpretation of this bond. The ELF analysis also demonstrates that the maximum function value for the monosynaptic basin of the I4 atom is directed towards the ELF minimum at the $\mathrm{I} 2$ atom (Figure $7 \mathrm{~B}$ ). 

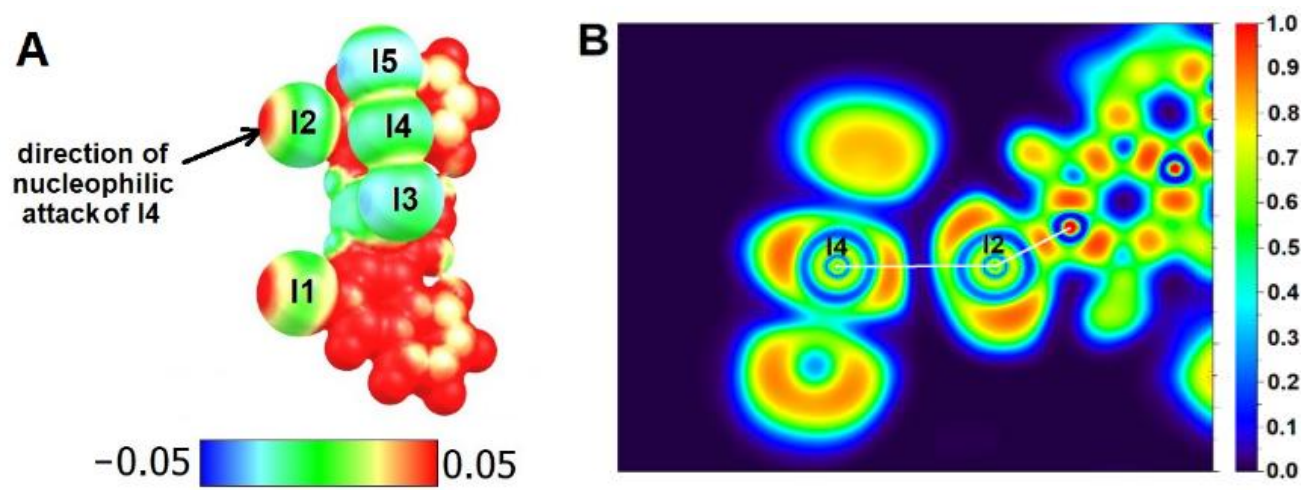

Figure 7. ESP distribution mapped on the $1.1 \times$ van der Waals surface for M1 (A) and ELF for $\mathrm{BM1}_{2}(\mathrm{~B})$.

The charge transfer (CT) plays only an insignificant role in these HaBs. The NBO analysis indicated that the main contribution in $\mathrm{CT}$ in $\mathrm{HaB}(\mathrm{I} \cdots \mathrm{I}) 1$ and $\mathrm{HaB}(\mathrm{I} \cdots \mathrm{I}) 2$ comes from the $\mathrm{LP}(\mathrm{I} 3) \rightarrow \sigma^{*}(\mathrm{I} 1-\mathrm{C})$ and $\mathrm{LP}(\mathrm{I} 4) \rightarrow \sigma^{*}(\mathrm{I} 2-\mathrm{C})$ transitions with the second order perturbation energies $\mathrm{E}(2)$ of only $1.3 \mathrm{kcal} / \mathrm{mol}$. Meanwhile, such CT confirmed the attribution of both these interactions to the type II HaBs with the I1 and I2 atoms serving as HaB donors; the back CT was not significant. The charge transfer in $\mathrm{HaB}(\mathrm{I} \cdots \mathrm{O}) 1-\mathrm{HaB}(\mathrm{I} \cdots \mathrm{O}) 3$ is even less substantial with $\mathrm{E}(2)$ of $0.8-0.9 \mathrm{kcal} / \mathrm{mol}$ for the predominant $\mathrm{LP}(\mathrm{O}) \rightarrow \sigma^{*}(\mathrm{I}-\mathrm{C})$ transitions and it is negligible for $\mathrm{HaB}(\mathrm{I} \cdots \mathrm{O}) 4$.

The interaction energies of the I...I and I ... O bonds were estimated using two approaches $\left(\mathrm{E}_{\text {int }}(\mathrm{BSSE})\right.$ and $\mathrm{E}_{\text {int }}(\mathrm{S})$; see Computational details). The $\mathrm{E}_{\text {int }}$ values of the individual bonds are within the range of -4.0 to $-10.4 \mathrm{kcal} / \mathrm{mol}$ that is typical for HaBs of weak to medium strength, with the I $\cdots$ I bonds being slightly more stable than the I $\cdots \mathrm{O}$ ones (Table 4).

\section{Discussion}

We developed a high-yielding facile route toward I-NON: This compound was formed via copper(II)-mediated iodination of $\mathrm{NON}$ with $\mathrm{I}_{2}$. Among various transition metal salts, copper(II) salts, in particular $\mathrm{Cu}(\mathrm{OAc})_{2} \cdot \mathrm{H}_{2} \mathrm{O}$, were found to be the best promotors of this reaction. The metal center played multiple roles in this iodination: Copper(II) stabilized NON in a deprotonated nitrosophenolate form by the coordination, thus preventing oxidative deoximation; the anionic aromatic $\mathrm{NON}-\mathrm{H}^{-}$form is preferable for aromatic electrophilic substitution involving such halogen as iodine. In addition, copper(II) activated $\mathrm{I}_{2}$ as an electrophilic agent [16]. Unlike $\mathrm{Cu}^{\mathrm{II}}$-mediated iodination of various aromatics, the NON iodination product was formed in the coordination sphere and, therefore, an additional step of the formed ligand liberation, by the reduction using $\mathrm{Na}_{2} \mathrm{~S}_{2} \mathrm{O}_{3}$, was required.

We also employed the prepared I-NON as a chelator and four new I-NON-based copper(II) complexes were obtained. The geometry of these complexes depends on the presence of I-NON ligands in deprotonated form and the ligand environment. Thus, complex 1 exhibited the cis- $(N, N)$,cis- $(O, O)$ geometry and complexes $\mathbf{3}$ and $\mathbf{4}$ were isolated as the trans- $(N, N)$,trans- $(O, O)$ isomers. The characteristic feature of the structures of $\mathbf{1}$ and 3 included intermolecular HaBs with the involvement of organoiodine atom of I-NON ligands. Thus, I-NON is a perspective new chelator, in which the presence of an iodine atom leads to the appearance of new structural features of I-NON-based complexes to give, for instance, HaBs in the solid state. Theoretical studies allowed the interpretation of the $\mathrm{I} \cdot$.. I and I...O short contacts in $\mathbf{1}$ and $\mathbf{3}$ as HaBs of weak-to-medium strength.

\section{Materials and Methods}

\subsection{Materials and Instrumentations}

The 1-Nitroso-2-naphthol, copper(II) salts, $\mathrm{I}_{2}, \mathrm{Na}_{2} \mathrm{~S}_{2} \mathrm{O}_{3}$, and solvents were obtained from a commercial source and used as received. The HRESI mass spectra were obtained 
on a Bruker micrOTOF spectrometer equipped with an electrospray ionization source and $\mathrm{MeOH}$ was employed as the solvent. The instrument was operated in positive ion mode using an $\mathrm{m} / \mathrm{z}$ range of 50-3000. The capillary voltage of the ion source was set at $-4500 \mathrm{~V}\left(\mathrm{ESI}^{+} \mathrm{MS}\right)$ and the capillary exit $\pm(70-150) \mathrm{V}$. In the isotopic pattern, the most intensive peak was reported. Infrared spectra were recorded using a Bruker FTIR TENSOR 27 instrument in KBr pellets. The absorption spectra were recorded on a Shimadzu UV 1800 spectrophotometer in MeCN. The ${ }^{1} \mathrm{H}$ and ${ }^{13} \mathrm{C}\left\{{ }^{1} \mathrm{H}\right\}$ NMR spectra were measured on a Bruker Avance III 400 spectrometer at ambient temperature. Residual solvent signals were used as the internal standard. Microanalyses $(\mathrm{C}, \mathrm{H}, \mathrm{N})$ were carried out on a Euro EA3028-HT instrument. The TGA studies were performed on a NETZSCH TG 209 F1 Libra thermoanalyzer and $\mathrm{MnO}_{2}$ powder was used as a standard. The initial weights of the samples were in the range of 1.1-2.3 $\mathrm{mg}$. The experiments were run in an open aluminum crucible in a stream of argon at a heating rate of $10 \mathrm{~K} / \mathrm{min}$. The final temperature was $610^{\circ} \mathrm{C}$. Processing of the thermal data was performed with Proteus analysis software. The powder diffraction experiments for 1 were carried out using D2Phaser diffractometer (Bruker), $\mathrm{Cu}$ anode, at $30 \mathrm{kV}$ and $10 \mathrm{~mA}$, and $\mathrm{CuK} \alpha_{1+2}$ radiation $\lambda_{\mathrm{CuK} \alpha 1}=1.54059 \AA$ and $\lambda_{\mathrm{CuK} \alpha 2}=1.54443 \AA$, from $6^{\circ}$ to $60^{\circ}$ on the $2 \theta$ scale at $1 \mathrm{~s} /$ step with step size $0.02^{\circ}$.

\subsection{X-ray Structure Determinations}

The XRD experiments were carried out using Oxford Diffraction "Xcalibur" diffractometer with monochromated $\mathrm{MoK} \alpha$ radiation. The crystals were thermostated at $100 \mathrm{~K}$ throughout the all-experiment time. The structures were solved by ShelXT [40] and Superflip [41] structure solution programs using Intrinsic Phasing and Charge Flipping methods, respectively, and refined using ShelXL [40] minimization program incorporated in Olex2 [42] program package. Empirical absorption correction was accounted by CrysAlisPro (Agilent Technologies, 2013) using spherical harmonics, implemented in SCALE3 ABSPACK scaling algorithm. The crystallographic data were deposited in the Cambridge Crystallographic Data Centre under the deposition codes CCDC 2076962-2076965 and can be obtained free of charge via the Internet, URL http:/ / www.ccdc.cam.ac.uk/structures / (accessed on 17 September 2021).

\subsection{Synthetic Work}

The 3-iodo-1-nitroso-2-naphthalenol. $\mathrm{Cu}(\mathrm{OAc})_{2} \cdot \mathrm{H}_{2} \mathrm{O}(28.9 \mathrm{mg}, 0.145 \mathrm{mmol})$, 1-nitroso2-naphthol (50 mg, $0.289 \mathrm{mmol})$, and iodine (293.6 mg, $1.16 \mathrm{mmol})$ were stirred at RT in $\mathrm{CHCl}_{3} / \mathrm{MeOH}(1 / 1 \mathrm{v} / \mathrm{v} ; 30 \mathrm{~mL})$ overnight. The solvent was then evaporated to dryness, and the residue formed was dissolved in THF $(30 \mathrm{~mL})$ and treated with a saturated aqueous solution of $\mathrm{Na}_{2} \mathrm{~S}_{2} \mathrm{O}_{3}(25 \mathrm{~mL})$. The organic layer was dried over $\mathrm{MgSO}_{4}$ and then the solvent was removed in vacuo at $30-35^{\circ} \mathrm{C}$ to give a crude product. Purification by column chromatography (silica gel, hexane/ethyl acetate 4:1, v/v) afforded I-NON (40.6 mg, $94 \%$ ) as a dark-brown powder. Alternatively, the product can be purified from NON by sublimation of the latter at $100{ }^{\circ} \mathrm{C}$ (0.07 torr).

Yield $40.6 \mathrm{mg}$, 94\%. Dark brown residue. Anal. Calcd for $\mathrm{C}_{10} \mathrm{H}_{6} \mathrm{NIO}_{2}$ : C, 40.16; $\mathrm{H}$, 2.02; N, 4.68. Found: C, 40.01; H, 1.95, N, 4.75\%. HRESI ${ }^{+}-\mathrm{MS}, m / z: 321.9300\left([\mathrm{M}+\mathrm{Na}]^{+}\right.$ requires 321.9341), $620.8707\left([2 \mathrm{M}+\mathrm{Na}]^{+}\right.$requires 620.8784). $v_{\max }(\mathrm{KBr}) / \mathrm{cm}^{-1}: 3434,3355$, $3176 \mathrm{~m} v(\mathrm{OH}) ; 2922 \mathrm{~m}, 2852 \mathrm{w} v(\mathrm{C}-\mathrm{H}) ; 1668 \mathrm{~s} v(\mathrm{C}=\mathrm{N}$ or $\mathrm{C}=\mathrm{O}) ; 1594 \mathrm{w}, 1541 \mathrm{~s} \delta\left(\mathrm{CH}_{\mathrm{Ar}}\right) .{ }^{1} \mathrm{H}$ NMR $\left(400 \mathrm{MHz}, \mathrm{CDCl}_{3}\right) \delta 17.16(\mathrm{~s}, 1 \mathrm{H}, \mathrm{NOH}), 8.40\left(\mathrm{~s}, 1 \mathrm{H}, \mathrm{HC}_{4}\right), 8.34(\mathrm{~d}, J=8.0 \mathrm{~Hz}, 1 \mathrm{H}$, $\left.\mathrm{HC}_{8}\right), 7.58\left(\mathrm{t}, J=7.5 \mathrm{~Hz}, 1 \mathrm{H}, \mathrm{HC}_{7}\right), 7.50\left(\mathrm{t}, J=7.4 \mathrm{~Hz}, 1 \mathrm{H}, \mathrm{HC}_{6}\right), 7.44(\mathrm{~d}, J=7.5 \mathrm{~Hz}, 1 \mathrm{H}$, $\left.\mathrm{HC}_{5}\right) .{ }^{13} \mathrm{C} \mathrm{NMR}\left(101 \mathrm{MHz}, \mathrm{CDCl}_{3}\right) \delta 177.93\left(\mathrm{C}_{2}\right), 156.62\left(\mathrm{C}_{4}\right), 143.03\left(\mathrm{C}_{1}\right), 131.55\left(\mathrm{C}_{7}\right), 130.97$ $\left(\mathrm{C}_{10}\right), 130.21\left(\mathrm{C}_{9}\right), 129.95\left(\mathrm{C}_{6}\right), 129.08\left(\mathrm{C}_{5}\right), 123.42\left(\mathrm{C}_{8}\right), 97.74\left(\mathrm{C}_{3}\right) .{ }^{13} \mathrm{C}$ ss-NMR $\delta 177.34\left(\mathrm{C}_{2}\right)$, $157.05\left(C_{4}\right), 140.49\left(C_{1}\right), 134.24\left(C_{7}\right), 132.63\left(C_{10}\right), 131.09\left(C_{9}, C_{6}\right), 130.11\left(C_{5}\right), 125.45\left(C_{8}\right)$, $104.24\left(\mathrm{C}_{3}\right)$. On heating in a capillary $\left(2^{\circ} / \mathrm{min}\right)$, this complex turns black and it decomposes at $154-156{ }^{\circ} \mathrm{C}$. TGA: $158-228{ }^{\circ} \mathrm{C}$ (mass loss $22.5 \%$ ), $228-576{ }^{\circ} \mathrm{C}$ (mass loss $37.6 \%$ ). Crystals suitable for XRD were obtained from the reaction mixture by its slow evaporation. 
[Cu(I-NON-H)(I-NON)](I $\left.\mathbf{I}_{3}\right)(\mathbf{1}) . \mathrm{Cu}\left(\mathrm{C}_{10} \mathrm{H}_{6} \mathrm{NO}_{2}\right)_{2}(0.025 \mathrm{mmol})$ was added to a solution of $\mathrm{I}_{2}(0.2 \mathrm{mmol})$ in $\mathrm{CHCl}_{3} / \mathrm{MeOH}(10 \mathrm{~mL}, 1 / 1 \mathrm{v} / \mathrm{v})$ placed in a $20-\mathrm{mL}$, round-bottomed flask. The reaction mixture was stirred at RT under ultrasonic treatment until the homogenization was complete and then left to stand at RT for slow evaporation. [Cu(I-NON-H)(I$\mathrm{NON})]\left(\mathrm{I}_{3}\right)(17 \mathrm{mg}, 67 \%)$ was released as black crystals after 7-9 d.

Yield $17 \mathrm{mg}, 67 \%$. Dark-brown-red crystals. Anal. Calcd for $\mathrm{C}_{20} \mathrm{H}_{11} \mathrm{CuI}_{5} \mathrm{~N}_{2} \mathrm{O}_{4}$ : C, 23.07; H, 1.06; N, 2.69. Found: C, 23.21; H, 1.19, N, 2.43\%. HRESI ${ }^{+}-\mathrm{MS}: m / z: 299.9514$ $\left([\mathrm{I}-\mathrm{NON}+\mathrm{H}]^{+}\right.$requires 299.9521), $321.9327\left([\mathrm{I}-\mathrm{NON}+\mathrm{Na}]^{+}\right.$requires 321.9341), 533.9123 $\left([\mathrm{Cu}(\mathrm{I}-\mathrm{NON}-\mathrm{H})(\mathrm{NON})]^{+}\right.$requires 533.9153$), 620.8766\left([2 \mathrm{I}-\mathrm{NON}+\mathrm{Na}]^{+}\right.$requires 620.8784$)$, $659.8088\left(\left[\mathrm{Cu}(\mathrm{I}-\mathrm{NON}-\mathrm{H})_{2}+\mathrm{H}\right]^{+}\right.$requires 659.8104$), 681.7916\left(\left[\mathrm{Cu}(\mathrm{I}-\mathrm{NON}-\mathrm{H})_{2}+\mathrm{Na}\right]^{+}\right.$ requires 681.7924), $1019.6751\left(\left[\mathrm{Cu}_{2}(\mathrm{I}-\mathrm{NON}-\mathrm{H})_{3}\right]^{+}\right.$requires 1019.6687). $\mathrm{HRESI}^{-} \mathrm{MS}, \mathrm{m} / z$ : $126.9059\left([\mathrm{I}]^{-}\right.$requires 126.9045$), 297.9354\left([\mathrm{I}-\mathrm{NON}-\mathrm{H}]^{-}\right.$requires 297.9365$), 380.7113\left(\left[\mathrm{I}_{3}\right]^{-}\right.$ requires 380.7134), $658.7954\left(\left[\mathrm{Cu}(\mathrm{I}-\mathrm{NON}-\mathrm{H})_{2}\right]^{-}\right.$requires 658.8026$), 956.7297([\mathrm{Cu}(\mathrm{I}-\mathrm{NON}-$ $\left.\mathrm{H})_{3}\right]^{-}$requires 956.7391). $v_{\max }(\mathrm{KBr}) / \mathrm{cm}^{-1}: 3443 \mathrm{~m} v(\mathrm{O}-\mathrm{H}) ; 3018 \mathrm{w} v\left(\mathrm{C}_{\mathrm{sp} 2}-\mathrm{H}\right) ; 1574 \mathrm{~m}-\mathrm{s}$ and $1537 \mathrm{~s} \vee(\mathrm{C}=\mathrm{N}, \mathrm{N}=\mathrm{O})$ and $\delta\left(\mathrm{CH}_{\mathrm{Ar}}\right)$. On heating in a capillary $\left(2^{\circ} / \mathrm{min}\right)$, this complex turns black and it decomposes at $189-190{ }^{\circ} \mathrm{C}$; this decomposition is accompanied with iodine elimination. TGA: $181-576{ }^{\circ} \mathrm{C}$ (weight loss $62.1 \%$ ). Crystals suitable for XRD were obtained on slow evaporation of the reaction mixture.

[Cu(I-NON-H) $)_{2}$ (2). $\mathrm{Cu}(\mathrm{OAc})_{2} \cdot \mathrm{H}_{2} \mathrm{O}(36.1 \mathrm{mg}, 0.18 \mathrm{mmol})$ and 3-iodo-1-nitroso-2naphthalenol (108 mg, $0.36 \mathrm{mmol})$ were refluxed in $\mathrm{MeOH}(30 \mathrm{~mL})$ for $3 \mathrm{~h}$. The formed black precipitate was filtered off, washed with methanol $(2 \times 10 \mathrm{~mL})$ and diethyl ether $(2 \times 10 \mathrm{~mL})$, and dried in air at RT to give the product $(77 \mathrm{mg}, 66 \%)$ as a black powder. Crystals suitable for XRD were obtained by slow evaporation of a THF solution at RT.

Yield $77 \mathrm{mg}, 66 \%$. Dark residue. Anal. Calcd for $\mathrm{C}_{20} \mathrm{H}_{10} \mathrm{CuI}_{2} \mathrm{~N}_{2} \mathrm{O}_{4}: \mathrm{C}, 36.42 ; \mathrm{H}$, 1.53; N, 4.25. Found: C, 36.57; H, 1.61, N, 4.32\%. HRESI ${ }^{+}-\mathrm{MS}, m / z: 533.9138$ ([Cu(I$\mathrm{NON}-\mathrm{H})(\mathrm{NON})]^{+}$requires 533.9153), $555.8969\left([\mathrm{Cu}(\mathrm{I}-\mathrm{NON}-\mathrm{H})(\mathrm{NON}-\mathrm{H})+\mathrm{Na}]^{+}\right.$requires 555.8957), $659.8121\left([\mathrm{M}+\mathrm{H}]^{+}\right.$requires 659.8104), $681.7948\left([\mathrm{M}+\mathrm{Na}]^{+}\right.$requires 681.7924$)$, $1340.5958\left([2 \mathrm{M}+\mathrm{Na}]^{+}\right.$requires 1340.5950). $v_{\max }(\mathrm{KBr}) / \mathrm{cm}^{-1}: 3060 \mathrm{w}, 2921 \mathrm{w} v(\mathrm{C}-\mathrm{H})$; $1576 \mathrm{~m}-\mathrm{w}$ and $1531 \delta\left(\mathrm{CH}_{\mathrm{Ar}}\right), 1511 \mathrm{~s} \delta\left(\mathrm{CH}_{\mathrm{Ar}}\right)$ and $v(\mathrm{~N}=\mathrm{O})$. On heating in a capillary $\left(2^{\circ} / \mathrm{min}\right)$, this complex turns black and it decomposes at $220-221^{\circ} \mathrm{C}$. TGA: $233-577^{\circ} \mathrm{C}$ (weight loss $48.8 \%$ ). Crystals of 3 suitable for XRD were obtained from solution of 2 in $\mathrm{CHCl}_{3} / \mathrm{THF}$ on its slow evaporation. Crystals of 4 suitable for XRD were obtained from a solution of 2 and excess $\mathrm{Py}$ in $\mathrm{CHCl}_{3} / \mathrm{MeOH}$ on its slow evaporation.

\subsection{Computational Details}

The calculations for the QTAIM, ESP, NBO, and IGM analyses were carried out using the crystallographic coordinates at the DFT level of theory with the PBE0 functional $[43,44]$ and the atom-pairwise dispersion correction with the Becke-Johnson damping scheme D3BJ [44,45]. The Gaussian 09 program package [46] was used. Cartesian $\mathrm{d}$ and $\mathrm{f}$ basis functions $(6 \mathrm{~d}, 10 \mathrm{f})$ were used in all calculations. The DZP-DKH basis set for non-iodine atoms [47-51] and the ADZP-DKH basis set for the iodine atoms were applied. The latter basis set was constructed from the DZP-DKH basis set by addition of diffuse functions taken from the ADZP basis set. The Douglas-Kroll-Hess second-order scalar relativistic correction (DKH) [52,53] was applied. An ultrafine integration grid was used for numerical integrations.

Five bimolecular clusters $\left(\mathbf{B M} \mathbf{1}_{1}, \mathbf{B M} \mathbf{1}_{2}, \mathbf{B M} \mathbf{3}_{1}, \mathbf{B M} \mathbf{3}_{2}\right.$, and $\left.\mathbf{B} \mathbf{M}_{3}\right)$ with geometries corresponding to the XRD structures of $\mathbf{1}$ and $\mathbf{3}$ and triplet spin state were used in the calculations. The $\mathrm{C}-\mathrm{H}$ bonds were fixed at $1.09 \AA$ and the $\mathrm{O}-\mathrm{H}$ bonds were fixed at $1.0 \AA$.

The structure of the monomeric complex M1 was optimized at the PBE0-D3BJ/DZP(IADZP) level and the energies and ESP values were refined at the PBE0-D3BJ/DZP-DKH(IADZP-DKH) level.

The topological analyses of the electron density distribution with the help of the AIM method of Bader [54] were performed using the program AIMAll [55] while the IGM analysis [56,57] was performed using the Multiwfn 3.8 [58] and VMD [59] software. The 
bond orbital nature was analyzed by using the natural bond orbital (NBO) partitioning scheme [60].

The interaction energies of the $\mathrm{I} \cdots \mathrm{I}$ and $\mathrm{I} \cdots \mathrm{O}$ bonds were estimated using two approaches. In the first one, the interaction energy $\left[\mathrm{E}_{\mathrm{int}}(\mathrm{BSSE})\right]$ was calculated as the difference of total energy of dimer and the sum of the energies of monomers with unrelaxed geometries with the corresponding basis set superposition error (BSSE) correction:

$$
\mathrm{E}_{\mathrm{int}}(\mathrm{BSSE})=(\mathrm{E}(\text { dimer })-\mathrm{E}(\text { monomer } 1)-\mathrm{E}(\text { monomer } 2)+\mathrm{BSSE}) / \mathrm{n}
$$

where $n=1$ for the I $\cdots \mathrm{I}$ bonds and $n=2$ for the I $\cdots$ O bonds. BSSE was estimated using the counterpoise method [61,62].

In the second approach, the interaction energy $\left[\mathrm{E}_{\text {int }}(\mathrm{S})\right]$ was calculated as the energy difference of two dimers:

$$
\mathrm{E}_{\text {int }}(\mathrm{S})=\mathrm{E}(\text { dimer2 })-\mathrm{E}(\text { dimer1 })
$$

In dimer1, the HaB donor I atom (I1 or I2) forming the halogen bond was substituted by the hydrogen atom and the length of this $\mathrm{C}-\mathrm{H}$ bond was fixed at $1.09 \AA$. In dimer2, the equivalent $\mathrm{I}$ atom not forming the $\mathrm{HaB}$ was replaced by the $\mathrm{H}$ atom (see Figure $\mathrm{S} 1$ in Supplementary Information for the structures of dimer1 and dimer2).

Supplementary Materials: The following are available online: scheme with tautomeric forms of 1-nitrosonaphthalene-2-ol; characterization of I-NON, 1 and 2; HRESI-MS, IR, UV-vis absorption, ${ }^{1} \mathrm{H}$ and ${ }^{13} \mathrm{C}\left\{{ }^{1} \mathrm{H}\right\}$ NMR spectra, TG and DTG curves, and powder and single-crystal $\mathrm{X}$-ray diffraction data for synthesized species; crystal data and structure refinement for I-NON, 1, 2, and 4; views of the fragment of molecular packing of I-NON, 1, and 3, demonstrating noncovalent contacts.

Author Contributions: Conceptualization, A.V.R., V.Y.K. and N.A.B.; methodology, A.V.R. and N.A.B.; investigation, Z.M.E., V.V.S. and M.L.K.; writing-original draft preparation, N.A.B. and M.L.K.; writing - review and editing, N.A.B., M.L.K. and V.Y.K.; visualization, N.A.B. and M.L.K.; supervision, N.A.B. and V.Y.K. All authors have read and agreed to the published version of the manuscript.

Funding: This study was funded by the Russian Science Foundation (19-13-00013; synthesis and characterization), the Russian Foundation for Basic Research (18-29-04006; XRD studies and crystal engineering) and the Fundação para a Ciência e a Tecnologia (FCT), Portugal, project UIDB/00100/2020 of Centro de Química Estrutural (theoretical calculations).

Institutional Review Board Statement: Not applicable.

Informed Consent Statement: Not applicable.

Data Availability Statement: Data is contained within the article and Supplementary materials. CIFs are available on www.ccdc.cam.ac.uk/data_request/cif (accessed on 17 September 2021).

Acknowledgments: We thank the Center for Magnetic Resonance, Center for X-ray Diffraction Studies, Thermogravimetric and Calorimetric Research Center, and Center for Chemical Analysis and Materials Research (all belonging to Saint Petersburg State University) for physicochemical studies.

Conflicts of Interest: The authors declare no conflict of interest. The funders had no role in the design of the study; in the collection, analyses, or interpretation of data; in the writing of the manuscript; or in the decision to publish the results.

\section{References}

1. Pike, S.J.; Heliot, A.; Seaton, C.C. ortho-Substituent effect on the crystal packing and solid state speciation of aromatic C-nitroso compounds. CrystEngComm 2020, 22, 5040-5048. [CrossRef]

2. Raić-Malić, S.; Tomašković, L.; Mrvoš-Sermek, D.; Prugovečki, B.; Cetina, M.; Grdiša, M.; Pavelić, K.; Mannschreck, A.; Balzarini, J.; De Clercq, E.; et al. Spirobipyridopyrans, spirobinaphthopyrans, indolinospiropyridopyrans, indolinospironaphthopyrans and indolinospironaphtho-1,4-oxazines: Synthesis, study of X-ray crystal structure, antitumoral and antiviral evaluation. Bioorg. Med. Chem. 2004, 12, 1037-1045. [CrossRef]

3. Ambrose, J.A. Fluorometric measurement of tyrosine in serum and plasma. Clin. Chem. 1974, 20, 505-510. [CrossRef] [PubMed] 
4. Knight, J.A.; Robertson, G.; Wu, J.T. The chemical basis and specificity of the nitrosonaphthol reaction. Clin. Chem. 1983, 29, 1969-1971. [CrossRef]

5. Fischer, V.; Mason, R.P. Formation of iminoxyl and nitroxide free radicals from nitrosonaphthols: An electron spin resonance study. Chem.-Biol. Interact. 1986, 57, 129-142. [CrossRef]

6. Tôei, K.; Motomizu, S.; Korenaga, T. Nitrosophenol and nitrosonaphthol derivatives as reagents for the spectrophotometric determination of iron and determination of micro-amounts in waters with 2-nitroso-5-dimethylaminophenol. Analyst 1975, 100, 629-636. [CrossRef]

7. Tôei, K.; Motomizu, S.; Yokosu, H. Extraction-spectrophotometric determination of nickel with 4-chloro-2-nitroso-1-naphthol and crystal violet. Anal. Chim. Acta 1979, 110, 329-334. [CrossRef]

8. Sirén, H.; Riekkola, M.L. Separation and determination of metals as complexes of 1-nitroso-2-naphthol-6-sulphonic and 2nitroso-1-naphthol-6-sulphonic acids-I: Ion-pair extraction and spectrophotometric method. Mikrochim. Acta 1986, 90, 159-173. [CrossRef]

9. Kitamori, T.; Suzuki, K.; Motojima, K.; Sawada, T.; Gohshi, Y. Determination of Sub-Part-per-Trillion Amounts of Cobalt by Extraction and Photoacoustic Spectroscopy. Anal. Chem. 1986, 58, 2275-2278. [CrossRef]

10. Memon, S.Q.; Bhanger, M.I.; Hasany, S.M.; Khuhawar, M.Y. The efficacy of nitrosonaphthol functionalized XAD-16 resin for the preconcentration/sorption of $\mathrm{Ni}(\mathrm{II})$ and $\mathrm{Cu}(\mathrm{II})$ ions. Talanta 2007, 72, 1738-1745. [CrossRef] [PubMed]

11. Tavana, J.; Edrisi, M. Synthesis of cobalt ferrite nanoparticles from thermolysis of prospective metal-nitrosonaphthol complexes and their photochemical application in removing methylene blue. Mater. Res. Express 2016, 3, 035009. [CrossRef]

12. Malcik, N.; Oktar, O.; Ozser, M.E.; Caglar, P.; Bushby, L.; Vaughan, A.; Kuswandi, B.; Narayanaswamy, R. Immobilised reagents for optical heavy metal ions sensing. Sens. Actuators B Chem. 1998, 53, 211-221. [CrossRef]

13. Basu, P.; Dey, T.K.; Ghosh, A.; Islam, S.M. Designing of a New Heterogeneous Polymer Supported Naphthyl-Azo Iron Catalyst for the Selective Oxidation of Substituted Methyl Benzenes. J. Inorg. Organomet. Polym. Mater. 2018, 28, 1158-1170. [CrossRef]

14. Marschalk, C. Halogen derivatives of 2-hydroxynaphthalene not substituted in the 1-position. Kuhlmann Villers-St. Paul. Bull. Société Chim. France 1928, 43, 1361-1367.

15. Issa, I.M.; Issa, R.M.; Amer, F.A.; Khattab, M.A. Spectrophotometric studies of some nitrosonaphthols. Indian J. Chem. Sect. A 1976, $14,713-714$.

16. Baird, W.C.; Surridge, J.H. Halogenation with copper(II) halides. Synthesis of aryl iodides. J. Org. Chem. 1970, 35, 3436-3442. [CrossRef]

17. Toshio, S.; Mitsushige, I.; Yoshiyuki, I.; Yoshinobu, T. Aromatic iodination with aluminum and copper(II) chlorides and iodine. Chem. Lett. 1982, 11, 1481-1484.

18. Akira, H.C.; Yasuo, S.J. A Convenient Procedure for Iodination of Electron-rich Aromatic Compounds using Iodine-Copper(II) Acetate. Bull. Chem. Soc. Jpn. 1984, 57, 2691-2692.

19. Saarinen, H.; Korvenranta, J. Crystal and molecular-structure of diacetone adduct of copper(II) complex of 1-Nitroso-2-Naphthol$\mathrm{CU}\left(\mathrm{C}_{10} \mathrm{H}_{6} \mathrm{NO}_{2}\right)_{2.2}\left(\mathrm{CH}_{3}\right)_{2} \mathrm{CO}$. Acta Chem. Scand. Ser. A-Phys. Inorg. Chem. 1975, 29, 409-413. [CrossRef]

20. Carugo, O.; Djinović, K.; Rizzi, M.; Castellani, C.B. Studies on metal complexes of ortho-quinone monooximes. Part 9. Analysis of the charge distribution within the o-quinone monooxime ligands through crystallographic data. J. Chem. Soc. Dalton Trans. 1991, 1255-1258. [CrossRef]

21. Cavallo, G.; Metrangolo, P.; Milani, R.; Pilati, T.; Priimagi, A.; Resnati, G.; Terraneo, G. The Halogen Bond. Chem. Rev. 2016, 116, 2478-2601. [CrossRef] [PubMed]

22. Cavallo, G.; Abate, A.; Rosati, M.; Paolo Venuti, G.; Pilati, T.; Terraneo, G.; Resnati, G.; Metrangolo, P. Tuning of Ionic Liquid Crystal Properties by Combining Halogen Bonding and Fluorous Effect. ChemPlusChem 2021, 86, 469-474. [CrossRef]

23. Taylor, M.S. Anion recognition based on halogen, chalcogen, pnictogen and tetrel bonding. Coord. Chem. Rev. 2020, 413, 213270. [CrossRef]

24. Scheiner, S.; Michalczyk, M.; Zierkiewicz, W. Coordination of anions by noncovalently bonded $\sigma$-hole ligands. Coord. Chem. Rev. 2020, 405, 213136. [CrossRef]

25. Riel, A.M.S.; Rowe, R.K.; Ho, E.N.; Carlsson, A.-C.C.; Rappé, A.K.; Berryman, O.B.; Ho, P.S. Hydrogen Bond Enhanced Halogen Bonds: A Synergistic Interaction in Chemistry and Biochemistry. Acc. Chem. Res. 2019, 52, 2870-2880. [CrossRef]

26. Wirth, S.; Rohbogner, C.J.; Cieslak, M.; Kazmierczak-Baranska, J.; Donevski, S.; Nawrot, B.; Lorenz, I.P. Rhodium(III) and iridium(III) complexes with 1,2-naphthoquinone-1-oximate as a bidentate ligand: Synthesis, structure, and biological activity. J. Biol. Inorg. Chem. 2010, 15, 429-440. [CrossRef]

27. Desiraju, G.R.; Ho, P.S.; Kloo, L.; Legon, A.C.; Marquardt, R.; Metrangolo, P.; Politzer, P.; Resnati, G.; Rissanen, K. Definition of the halogen bond (IUPAC Recommendations 2013). Pure Appl. Chem. 2013, 85, 1711. [CrossRef]

28. Osman, A.M.; Khalaf, A.A.; Amer, F.A. Synthesis of some naphthoxazoles from nitrosonaphthols. Indian J. Chem. 1974, 12, 120-123.

29. Zincke, T.; Schmunk, L. Ueber die Einwirkung von Chlor auf Chinon0xime (Nitrosophenole). Justus Liebigs Ann. Chem. 1890, 257, 133-155. [CrossRef]

30. Bolotin, D.S.; Bokach, N.A.; Demakova, M.Y.; Kukushkin, V.Y. Metal-Involving Synthesis and Reactions of Oximes. Chem. Rev. 2017, 117, 13039-13122. [CrossRef] [PubMed] 
31. Orpen, A.G.; Brammer, L.; Allen, F.H.; Kennard, O.; Watson, D.G.; Taylor, R. Supplement. Tables of bond lengths determined by X-ray and neutron diffraction. Part 2. Organometallic compounds and co-ordination complexes of the d-and f-block metals. J. Chem. Soc. Dalton Trans. 1989, S1-S83. [CrossRef]

32. Bikbaeva, Z.M.; Ivanov, D.M.; Novikov, A.S.; Ananyev, I.V.; Bokach, N.A.; Kukushkin, V.Y. Electrophilic-Nucleophilic Dualism of Nickel(II) toward $\mathrm{Ni} \cdots$ I Noncovalent Interactions: Semicoordination of Iodine Centers via Electron Belt and Halogen Bonding via $\sigma$-Hole. Inorg. Chem. 2017, 56, 13562-13578. [CrossRef] [PubMed]

33. Bondi, A. van der Waals Volumes and Radii. J. Phys. Chem. 1964, 68, 441-451. [CrossRef]

34. Bondi, A. van der Waals Volumes and Radii of Metals in Covalent Compounds. J. Phys. Chem. 1966, 70, 3006-3007. [CrossRef]

35. Batsanov, S.S. Van der Waals Radii of Elements. Inorg. Mater. 2001, 37, 871-885. [CrossRef]

36. Wang, Y.-H.; Lu, Y.-X.; Zou, J.-W.; Yu, Q.-S. Theoretical investigation on charge-assisted halogen bonding interactions in the complexes of bromocarbons with some anions. Int. J. Quantum Chem. 2008, 108, 90-99. [CrossRef]

37. Grabowski, S.J.; Bilewicz, E. Cooperativity halogen bonding effect-Ab initio calculations on $\mathrm{H}_{2} \mathrm{CO} \cdots(\mathrm{ClF}) \mathrm{n}$ complexes. Chem. Phys. Lett. 2006, 427, 51-55. [CrossRef]

38. Lu, Y.-X.; Zou, J.-W.; Wang, Y.-H.; Jiang, Y.-J.; Yu, Q.-S. Ab Initio Investigation of the Complexes between Bromobenzene and Several Electron Donors: Some Insights into the Magnitude and Nature of Halogen Bonding Interactions. J. Phys. Chem. A 2007, 111, 10781-10788. [CrossRef]

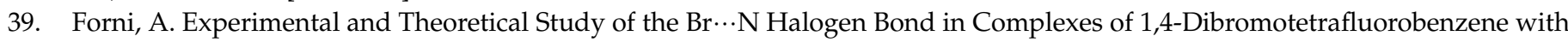
Dipyridyl Derivatives. J. Phys. Chem. A 2009, 113, 3403-3412. [CrossRef]

40. Sheldrick, G. SHELXT-Integrated space-group and crystal-structure determination. Acta Crystallogr. Sect. A 2015, 71, 3-8. [CrossRef]

41. Palatinus, L.; Chapuis, G. SUPERFLIP-a computer program for the solution of crystal structures by charge flipping in arbitrary dimensions. J. Appl. Crystallogr. 2007, 40, 786-790. [CrossRef]

42. Dolomanov, O.V.; Bourhis, L.J.; Gildea, R.J.; Howard, J.A.K.; Puschmann, H. OLEX2: A complete structure solution, refinement and analysis program. J. Appl. Crystallogr. 2009, 42, 339-341. [CrossRef]

43. Adamo, C.; Barone, V. Toward reliable density functional methods without adjustable parameters: The PBE0 model. J. Chem. Phys. 1999, 110, 6158-6170. [CrossRef]

44. Grimme, S.; Ehrlich, S.; Goerigk, L. Effect of the damping function in dispersion corrected density functional theory. J. Comput. Chem. 2011, 32, 1456-1465. [CrossRef]

45. Grimme, S.; Antony, J.; Ehrlich, S.; Krieg, H. A consistent and accurate ab initio parametrization of density functional dispersion correction (DFT-D) for the 94 elements H-Pu. J. Chem. Phys. 2010, 132, 154104. [CrossRef] [PubMed]

46. Frisch, M.J.; Trucks, G.W.; Schlegel, H.B.; Scuseria, G.E.; Robb, M.A.; Cheeseman, J.R.; Scalmani, G.; Barone, V.; Mennucci, B.; Petersson, G.A.; et al. Gaussian 09, Revision D.01; Gaussian: Wallingford, CT, USA, 2013.

47. Jorge, F.E.; Neto, A.C.; Camiletti, G.G.; Machado, S.F. Contracted Gaussian basis sets for Douglas-Kroll-Hess calculations: Estimating scalar relativistic effects of some atomic and molecular properties. J. Chem. Phys. 2009, 130, 064108. [CrossRef]

48. Canal Neto, A.; Muniz, E.P.; Centoducatte, R.; Jorge, F.E. Gaussian basis sets for correlated wave functions. Hydrogen, helium, first- and second-row atoms. J. Mol. Struct. THEOCHEM 2005, 718, 219-224. [CrossRef]

49. Camiletti, G.G.; Machado, S.F.; Jorge, F.E. Gaussian basis set of double zeta quality for atoms K through Kr: Application in DFT calculations of molecular properties. J. Comput. Chem. 2008, 29, 2434-2444. [CrossRef]

50. Barros, C.L.; de Oliveira, P.J.P.; Jorge, F.E.; Canal Neto, A.; Campos, M. Gaussian basis set of double zeta quality for atoms Rb through Xe: Application in non-relativistic and relativistic calculations of atomic and molecular properties. Mol. Phys. 2010, 108, 1965-1972. [CrossRef]

51. Pritchard, B.P.; Altarawy, D.; Didier, B.; Gibson, T.D.; Windus, T.L. New Basis Set Exchange: An Open, Up-to-Date Resource for the Molecular Sciences Community. J. Chem. Inf. Model. 2019, 59, 4814-4820. [CrossRef]

52. Douglas, M.; Kroll, N.M. Quantum electrodynamical corrections to the fine structure of helium. Ann. Phys. 1974, 82, 89-155. [CrossRef]

53. Hess, B.A. Applicability of the no-pair equation with free-particle projection operators to atomic and molecular structure calculations. Phys. Rev. A 1985, 32, 756-763. [CrossRef] [PubMed]

54. Bader, R.F.W. Atoms in Molecules: A Quantum Theory; Oxford University Press: Oxford, UK, 1990.

55. Keith, T.A.; Gristmill, T.K. AIMAll; Gristmill Software: Overland Park, KS, USA, 2014.

56. Lefebvre, C.; Rubez, G.; Khartabil, H.; Boisson, J.-C.; Contreras-García, J.; Hénon, E. Accurately extracting the signature of intermolecular interactions present in the NCI plot of the reduced density gradient versus electron density. Phys. Chem. Chem. Phys. 2017, 19, 17928-17936. [CrossRef] [PubMed]

57. Lefebvre, C.; Khartabil, H.; Boisson, J.-C.; Contreras-García, J.; Piquemal, J.-P.; Hénon, E. The Independent Gradient Model: A New Approach for Probing Strong and Weak Interactions in Molecules from Wave Function Calculations. ChemPhysChem 2018, 19, 724-735. [CrossRef] [PubMed]

58. Lu, T.; Chen, F. Multiwfn: A multifunctional wavefunction analyzer. J. Comput. Chem. 2012, 33, 580-592. [CrossRef]

59. Humphrey, W.; Dalke, A.; Schulten, K. VMD: Visual molecular dynamics. J. Mol. Graph. 1996, 14, 33-38. [CrossRef]

60. Reed, A.E.; Curtiss, L.A.; Weinhold, F. Intermolecular interactions from a natural bond orbital, donor-acceptor viewpoint. Chem. Rev. 1988, 88, 899-926. [CrossRef] 
61. Boys, S.F.; Bernardi, F. The calculation of small molecular interactions by the differences of separate total energies. Some procedures with reduced errors. Mol. Phys. 1970, 19, 553-566. [CrossRef]

62. Simon, S.; Duran, M.; Dannenberg, J.J. How does basis set superposition error change the potential surfaces for hydrogen-bonded dimers? J. Chem. Phys. 1996, 105, 11024-11031. [CrossRef] 